\title{
Comparison of Digital Breast Tomosynthesis and Digital Mammography for Detection of Breast Cancer in Kuwaiti Women
}

\author{
Akram M. Asbeutah ${ }^{a}$ Nouralhuda Karmani ${ }^{b}$ AbdulAziz A. Asbeutah ${ }^{c}$ \\ Yasmin A. Echreshzadeh ${ }^{b}$ Abdullah A. AlMajran ${ }^{d}$ Khalid H. Al-Khalifah ${ }^{a}$ \\ ${ }^{a}$ Department of Radiologic Sciences, Faculty of Allied Health Sciences, Kuwait University, Kuwait City, Kuwait; \\ ${ }^{b}$ Department of Clinical Radiology, Clinical Breast Imaging Unit, Al-Sabah Hospital, Ministry of Health, Kuwait

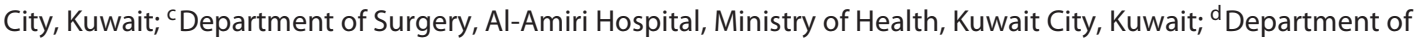 \\ Community Medicine and Behavioral Sciences, Faculty of Medicine, Kuwait University, Kuwait City, Kuwait
}

\section{Significance of the Study}

- Breast cancer is ranked as the most common malignancy among Arab and Kuwaiti women and represents $38 \%$ of all cancer cases. Digital breast tomosynthesis (DBT) is the emerging technique for imaging breast lesions. DBT improves the clinical accuracy of mammography due to improved image quality. This could have a positive impact on the mortality rate.

\section{Keywords}

Breast cancer · Digital breast tomosynthesis · Digital mammography $\cdot$ Histopathology

\begin{abstract}
Objective: To investigate the sensitivity and specificity of digital mammography (DM) and digital breast tomosynthesis (DBT) for the detection of breast cancer in comparison to histopathology findings. Subjects and Methods: We included 65 breast lesions in 58 women, each detected by two diagnostic mammography techniques - DM and DBT using Senographe Essential (GE Healthcare, Buc, France) - and subsequently confirmed by histopathology. The Breast Imaging Reporting and Data System was used for characterizing the lesions. Results: The average age of women was 48.3 years (range 26-81 years). There were 34 malignant and 31
\end{abstract}

benign breast lesions. The sensitivity of DM and DBT was 73.5 and $100 \%$, respectively, while the specificity was 67.7 and 94\%, respectively. Receiver operating characteristic curve analysis showed an overall diagnostic advantage of DBT over DM, with a significant difference between DBT and DM $(p<0.001)$. By performing Cohen's kappa test, we found that there was a strong level of agreement according to Altman guidelines between DBT and histopathology findings (0.97), but a weak agreement between DM and histopathology findings (0.47). Conclusion: DBT improves the clinical accuracy of mammography by increasing both sensitivity and specificity. We believe that this improvement is due to improved image visibility and quality. These results could be of interest to health care institutions as they may impact their decision on whether to upgrade to DBT not only for diagnosis, but also for screening.

(C)2018 The Author(s) Published by S. Karger AG, Basel

\begin{tabular}{ll}
\hline KARGER & $\begin{array}{l}\text { (2018 The Author(s) } \\
\text { Published by S. Karger AG, Basel }\end{array}$ \\
E-Mail karger@karger.com & $\begin{array}{l}\text { This is an Open Access article licensed under the Creative Commons } \\
\text { Attribution-NonCommercial-4.0 International License (CC BY-NC) } \\
\text { (http://www.karger.com/Services/OpenAccessLicense), applicable to } \\
\text { the online version of the article only. Usage and distribution for } \\
\text { commercial purposes requires written permission. }\end{array}$
\end{tabular}

Dr. Akram M. Asbeutah

Department of Radiologic Sciences, Faculty of Allied Health Sciences

Kuwait University, P.O. Box 31470

Sulaibikhat 90805 (Kuwait)

E-Mail asbeutah_akram@ @otmail.com 


\section{Introduction}

Breast cancer is the most common malignancy among women worldwide with increasing incidence rates [1]. It was ranked as the most common malignancy among Arab women, constituting $14-42 \%$, and represents $38 \%$ of all cancer cases among Kuwaiti women [2]. Breast cancer among women living in the Middle East occurs almost 10 years earlier than in the United States of America and Europe [3]. Moreover, $50 \%$ of all patients in Arab countries are below the age of 50 years, while only $25 \%$ of patients in industrialized nations are below the age of 50 years [4].

Digital mammography (DM) is the preferred breast imaging technique for diagnostic and/or screening purposes [5] and is the most commonly used breast imaging technique in Kuwait. Nevertheless, the limitations of mammography are well known [3]. The sensitivity of mammography in dense breast tissue can be limited due to the presence of overlapping fibroglandular tissue that reduces conspicuity of abnormalities [6]. Another factor influencing cancer visibility is the pattern of tumor growth. Tumors that do not produce a mass are often difficult to detect on mammography [7]. Depending on breast density and tumor growth pattern, the false-negative rate of mammography ranges from 8 to $66 \%$ in symptomatic women [8]. Solutions to these problems include ultrasound (US) and magnetic resonance imaging (MRI), but they are not the optimal solution because despite US being safe and inexpensive, it has been reported to be operator-dependent with low interobserver agreement, particularly for small malignancies [9]. Moreover, despite MRI being safe and sensitive [10], it is expensive and time-consuming and in most cases not available in the same department, with a low-to-moderate specificity ranging from 37 to $97 \%$ [11].

Recent advances in digital imaging in general, and DM in particular, have led to the development of digital breast tomosynthesis (DBT) systems for three-dimensional breast examinations by taking multiple low-dose images per view along an arc over the breast [12]. By combining information from different projections, DBT improves the differentiation of the adjacent anatomical structures, alleviating the superimposition effect [13]. More recent studies indicate about $30 \%$ increased DBT sensitivity and specificity compared with DM [14], with a $40 \%$ reduction in callback in screening [15-18]. DBT has been implemented in Kuwait during the last 5 years. It is used by radiologists only for further clarification of a suspicious breast lesion which is not seen very well by DM alone. DBT is not used as a screening technique in Kuwait. Sev- eral studies have compared DM to DBT in Western women. Apart from one study done in Egypt [19], there is a lack of studies using DBT compared to DM in Kuwaiti women.

To the best of our knowledge, this is the first study in the Arabian Gulf to investigate the sensitivity and specificity of DBT compared to DM for the detection of breast cancer and to compare the mammography results to histopathology-proven breast lesions in a series of Kuwaiti women.

\section{Subjects and Methods}

Subjects

Participants included women attending the Clinical Breast Imaging Unit of our hospital, women referred from other clinics/hospitals for a palpable breast lump on clinical examination, or women seeking a second opinion after suspicious findings on US examination or abnormal screening mammography during the period of September 2017 to March 2018. The exclusion criteria comprised women with the presence of breast implants, women who were pregnant, and women who were lactating.

\section{$D M$ and DBT Image Acquisition}

A dedicated DM unit, Senographe Essential (GE Healthcare, Buc, France), was used for imaging. The system supported both DM and DBT imaging by acquiring craniocaudal and mediolateral oblique projections during the same breast compression (25$30 \mathrm{kV}, 100 \mathrm{~mA}$, amorphous silicon/cesium iodide material detector $24 \times 31 \mathrm{~cm}$, pixel size $100 \mu \mathrm{m}$, dual target material of molybdenum/rhodium X-ray tube, rhodium/molybdenum filters, and 5:1 antiscatter grid). DBT images were acquired with X-ray tube rotation through the angular range of $25^{\circ}$ with nine projections with the breast in standard compression in craniocaudal and/or mediolateral oblique projections. Image acquisition was performed with a step-and-shoot method, with an acquisition time of $<10 \mathrm{~s}$ for one breast. Iterative image reconstruction was performed immediately after image acquisition with slice thickness of $0.5 \mathrm{~mm}$ and time of reconstruction of $<15 \mathrm{~s}$. The Picture Archiving and Communication System was used to access images from the two imaging modalities. Both DM and DBT were performed by one licensed radiographer with more than 10 years of experience in breast imaging.

\section{DM and DBT Image and Tissue Analysis}

The double image reading system was used for DM and DBT image interpretation. One experienced radiologist with more than 10 years of experience in breast imaging interpreted the DM and DBT images. Moreover, a consultant radiologist with more than 15 years of experience in breast imaging approved the diagnosis. DM and DBT images were reviewed separately on dedicated workstations with two high-resolution display monitors to determine the presence or absence of any abnormal findings. The DM views were evaluated first, then the images obtained by DBT were reviewed in cine mode in manual scroll step. No comparative examinations or other clinical information about the subjects was provided in the interpretation sessions. All detected lesions were rated according to the American College of Radiology (ACR) Breast Imaging Reporting and Data System (BI-RADS)-based 
Table 1. Types of breast lesions according to histopathological findings

\begin{tabular}{lc}
\hline Type of tumor & Number \\
\hline Malignant lesions & 34 \\
Lobular invasive carcinoma & 3 \\
Invasive ductal carcinoma & 29 \\
Invasive and ductal carcinoma in situ & 2 \\
\hline Benign lesions & 31 \\
Fibroadenoma & 6 \\
Fibrocystic changes & 4 \\
Diffuse cellular stroma & 6 \\
Granulomatous inflammation & 4 \\
Microcalcification & 3 \\
Postsurgical scar & 2 \\
Ductal papilloma & 2 \\
Ductal cell hyperplasia & 2 \\
Sclerosing adenosis and duct ectasia & 1 \\
Fat necrosis & 1 \\
\hline
\end{tabular}

Table 2. Comparison of DM and DBT results with histopathological findings

\begin{tabular}{lccl}
\hline Imaging procedure & \multicolumn{2}{l}{ Histopathology } \\
\cline { 2 - 4 } & malignant & benign & total \\
\hline DM & 25 & 12 & 37 \\
$\quad$ Malignant & 9 & 19 & 28 \\
$\quad$ Benign & 33 & 1 & 34 \\
DBT & 1 & 30 & 31 \\
$\quad$ Malignant & 34 & 31 & 65 \\
\hline Benign & & & \\
\hline Total &
\end{tabular}

DBT, digital breast tomosynthesis; DM, digital mammography.

scale. BI-RADS 1-3 were considered benign, BI-RADS 4 as suspicious for malignancy, and BI-RADS 5 and 6 were considered malignant [20]. The malignant features included focal density, architectural distortion, ill-defined margins of the mass, and a cluster of microcalcifications, or a combination of these. The radiologist decided whether the margins of a mass were clearly more visible with DM or DBT and rated which was superior. The ACR BIRADS category of each lesion, of each diagnostic method, was compared with the histopathology findings. The final diagnosis was established by histopathological analysis of core biopsies, vacuum-assisted stereotactic biopsy, or surgical excision of the specimen. Histopathological analysis was done at the Department of Pathology.

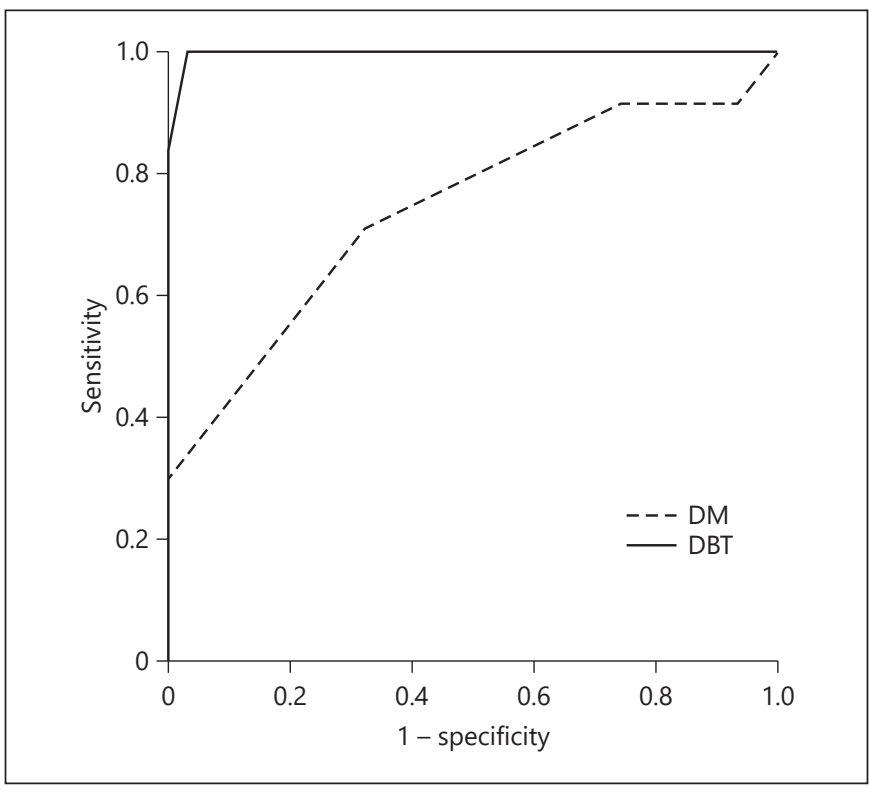

Fig. 1. ROC curves for breast DM and DBT according to the BIRADS classification. BI-RADS, Breast Imaging Reporting and Data System; DBT, digital breast tomosynthesis; DM, digital mammography; ROC, receiver operating characteristic.

\section{Statistical Analysis}

Data were entered into Excel 2013. All statistical analyses were carried out using the Statistical Package for the Social Sciences (SPSS) version 25 for Windows (SPSS Inc., Chicago, IL, USA). The results of the two imaging modalities (DM and DBT) were compared with the histopathology report. Specificity, sensitivity, and predictive values were processed. A receiver operating characteristic (ROC) curve was generated for each diagnostic method. The options in the SPSS package were used for the calculation and presentation of the ROC curve in accordance with the ACR BI-RADS classification. Cohen's kappa test was used for the evaluation of agreement between each diagnostic method and the histopathology findings. Interpretation of the ROC curves was done as described in Park et al. [21]. Cohen's kappa coefficient was interpreted based on Altman guidelines [22].

\section{Results}

The average age \pm standard deviation of all subjects was $48.30 \pm 12.90$ years (range $26-81$ years). Using DM and DBT, we evaluated imaging features of 65 breast lesions in 58 subjects and compared them to histopathology findings. The dataset consisted of 34 malignancies and 31 benign breast lesions (Table 1).

Sensitivity, specificity, predictive values, and ROC curves for the two diagnostic methods were analyzed based on the information obtained from Table 2. The sen-
12

Med Princ Pract 2019;28:10-15

DOI: $10.1159 / 000495753$
Asbeutah/Karmani/Asbeutah/

Echreshzadeh/AlMajran/Al-Khalifah 
Fig. 2. A 49-year-old patient. a DM mediolateral oblique projection of the left breast showed a large irregular dense mass with calcification involving the whole upper outer quadrant (circle), classified as BIRADS 4. Also note the calcified fibroadenoma in the left upper outer quadrant (arrow). b DBT mediolateral oblique projection of the left breast showed ill-defined mass margins (circle), classified as BIRADS 5. Histopathology confirmed this to be invasive ductal carcinoma. BI-RADS, Breast Imaging Reporting and Data System; DBT, digital breast tomosynthesis; DM, digital mammography.
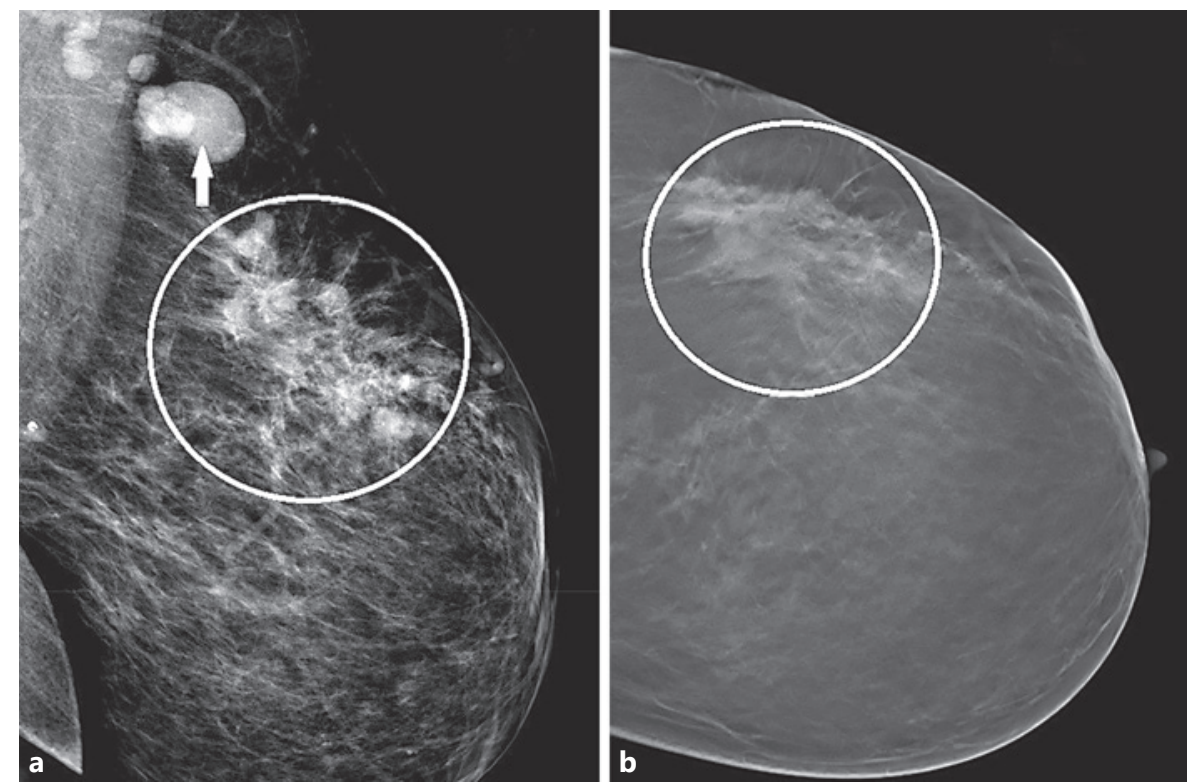

sitivity and specificity were 73.5 and $67.7 \%$ for DM and 100 and $94 \%$ for DBT, respectively. The positive predictive value was 0.970 for $\mathrm{DBT}$ and 0.714 for DM. The negative predictive value was also higher in DBT (1.00) than in DM (0.70).

We compared the area under the curve for the two analyzed diagnostic methods (Figure 1). The greatest area was under the DBT curve (0.984) and the smallest area under the DM curve (0.706). By comparing the ROC curves, we showed that there was a statistically significant difference between the diagnostic accuracy of DBT and DM $(p<0.001)$. Most of the malignant lesions had illdefined borders (Fig. 2), with $73 \%$ in DM and $97 \%$ in DBT.

Cohen's kappa test was used to test for the level of agreement between DM and DBT and histopathology findings. There was a weak level of agreement according to Altman guidelines [22] between DM and histopathology findings (0.47), while there was a strong level of agreement between DBT and histopathology findings (0.97).

\section{Discussion}

This study was undertaken to determine the sensitivity and specificity with predictive values for DBT at the Clinical Breast Imaging Unit of our hospital. DBT was approved by the Food and Drug Administration in 2011, and since then it has been developed in an effort to im- prove the accuracy of breast imaging by creating a threedimensional mammogram, virtually eliminating areas that obscure the breast parenchyma.

DM has a significantly lower sensitivity and specificity than DBT in diagnosing malignant breast lesions. The sensitivity of DBT was $100 \%$ in this study, meaning that DBT detected all of the malignant tumors. This factor alone is a significant indication of the superiority of DBT over DM. Furthermore, ROC curve analysis showed an overall diagnostic advantage of DBT over DM, clearly seen when comparing ROC curves for the two analyzed diagnostic methods (Fig. 1). This study showed a high sensitivity and specificity of DBT over DM. This advantage of DBT over DM provides two main benefits, namely increased rates of cancer detection and reduced callback rates $[15-18,23]$.

Lesions were easier to distinguish on DBT, and the analysis of margins was also more precise. In several cases, superimposed breast tissue caused false-positive findings on mammography that were later reported as normal breast tissue on DBT. The increased sensitivity and specificity in this study are due to improved image quality in DBT over DM in terms of spatial and contrast resolutions. Factors that improved spatial resolution include a thin tomographic slice acquisition $(0.5 \mathrm{~mm})$, an excellent amorphous detector material made of silicon/cesium iodide, a pixel size of $100 \mu \mathrm{m}$, and a voxel depth of 14 bits of gray shades on the imaging system. Additionally breast compression, which serves to achieve immobilization and to further reduce the radiation dose by reducing 
breast thickness, was used. However, contrast resolution was achieved by the use of a proper range of $25-30 \mathrm{kV}$ during the procedure, a molybdenum/molybdenum target/filter material, 5:1 antiscatter grid, and breast compression [24]. Also, due to the basic principle of DBT which reduces anatomical noise, sensitivity and specificity were increased, thus the lesions were better visualized and more accurately categorized according to the ACR BI-RADS criteria.

Even though one radiologist interpreted the mammographic results and one radiographer performed the two procedures, possible causes of false-positive and falsenegative results in this study could be attributed to poor positioning, perception error, incorrect interpretation of a suspect finding, subtle features of malignancy, and slow growth of a lesion [24]. Thus, in symptomatic patients with a negative mammography, additional diagnostic workup using another breast imaging modality is advised.

Although DBT has many advantages, there are also a few disadvantages. These include the possibility that some of the malignances may be missed or misinterpreted [25], the limited capability of visualization of microcalcification [26], and increased radiation dose when DBT is combined with DM [27]. The increased total dose is still below the Food and Drug Administration safety limits of $3 \mathrm{mGy} / \mathrm{view}$. Radiation dose is cumulative over time, so it is prudent to limit exposure to DBT. A further dose reduction can be achieved by using a target/filter combination of tungsten/rhodium as suggested recently [28]. As we mentioned previously, DBT has already been implemented in Kuwait for the last 5 years. Also, it is used by radiologists only to obtain more clarification of a suspicious breast lesion following DM to obtain a better image quality for better diagnosis. In addition, DBT is not used as a screening technique in Kuwait. Despite these disadvantages, researchers are exploring the possibilities of effectively using it in screening [29]. Moreover, clinical studies indicate that contrast-enhanced DBT gives results that are concordant with MRI, increasing the diagnostic accuracy of DBT [30].

The limitations of this study are its small sample size and the fact that it involved a nonblinded review. We recommend further studies with a larger population of Arab women residing in different Arab countries in order to confirm or refute our findings. In addition, further research is needed to explore the optimal imaging technique that will give the best image quality with the least radiation dose to the patient. This aims for better patient diagnosis, improving further patient management. Final- ly, additional research is needed to determine whether DBT can replace DM as a diagnostic technique and whether early detection of breast cancer using DBT is associated with a decrease in mortality rate.

\section{Conclusion}

DBT improves the clinical accuracy of mammography by increasing both sensitivity and specificity, possibly due to improved image visibility and quality. These data could be of great interest to health care institutions as they may impact their decision on whether to upgrade to DBT not only for diagnosis, but also for screening.

\section{Acknowledgments}

We thank our subjects for their participation in this study. We also thank the Clinical Breast Imaging Unit of our hospital for their support and help. Special appreciation goes to the Department of Histopathology for allowing us to access the subjects' histopathology reports.

\section{Statement of Ethics}

The study protocol was approved by the combined Ethics Committee of the Health Sciences Centre and Ministry of Public Health, Kuwait. Informed consent was obtained from the women who participated in this study.

\section{Disclosure Statement}

The authors declare no conflicts of interest.

References

1 Khalil AM, Ayad EE, El-Sheikh SA. Immunohistochemical expression of c-kit in invasive breast carcinoma of different nuclear grades. Med J Cairo Univ. 2012;80:345-51.

2 Al Ramadhan MA. Eradicating Breast Cancer: Longevity Impact on Kuwaiti Women. Asian Pac J Cancer Prev. 2017 Mar;18(3): 803-9.

3 American Cancer Society. Cancer Facts and Figures 2015. Atlanta (GA): American Cancer Society; 2015.

4 Saadat S. Can we prevent breast cancer? Int J Health Sci (Qassim). 2008 Jul;2(2):167-70.
Med Princ Pract 2019;28:10-15 DOI: $10.1159 / 000495753$
Asbeutah/Karmani/Asbeutah/ Echreshzadeh/AlMajran/Al-Khalifah 
5 Cheung YC, Lin YC, Wan YL, Yeow KM, Huang PC, Lo YF, et al. Diagnostic performance of dual-energy contrast-enhanced subtracted mammography in dense breasts compared to mammography alone: interobserver blind-reading analysis. Eur Radiol. 2014 Oct;24(10):2394-403.

6 Chan HP, Wei J, Sahiner B, Rafferty EA, Wu $\mathrm{T}$, Roubidoux MA, et al. Computer-aided detection system for breast masses on digital tomosynthesis mammograms: preliminary experience. Radiology. 2005 Dec;237(3):107580.

7 Andersson I, Ikeda DM, Zackrisson S, Ruschin M, Svahn T, Timberg P, et al. Breast tomosynthesis and digital mammography: a comparison of breast cancer visibility and BIRADS classification in a population of cancers with subtle mammographic findings. Eur Radiol. 2008 Dec;18(12):2817-25.

8 Teertstra HJ, Loo CE, van den Bosch MA, van Tinteren H, Rutgers EJ, Muller SH, et al. Breast tomosynthesis in clinical practice: initial results. Eur Radiol. 2010 Jan;20(1):16-24.

9 Abdullah N, Mesurolle B, El-Khoury M, Kao E. Breast Imaging Reporting and Data System lexicon for US: interobserver agreement for assessment of breast masses. Radiology. 2009 Sep;252(3):665-72.

10 Orel SG, Schnall MD. MR imaging of the breast for the detection, diagnosis, and staging of breast cancer. Radiology. 2001 Jul; 220(1):13-30.

11 Esserman L, Wolverton D, Hylton N. Magnetic resonance imaging for primary breast cancer management: current role and new applications. Endocr Relat Cancer. 2002 Jun; 9(2):141-53.

12 Alakhras M, Bourne R, Rickard M, Ng KH, Pietrzyk M, Brennan PC. Digital tomosynthesis: a new future for breast imaging? Clin Radiol. 2013 May;68(5):e225-36.
13 Kontos D, Bakic PR, Carton AK, Troxel AB, Conant EF, Maidment AD. Parenchymal texture analysis in digital breast tomosynthesis for breast cancer risk estimation: a preliminary study. Acad Radiol. 2009 Mar;16(3): 283-98.

14 Roganovic D, Djilas D, Vujnovic S, Pavic D, Stojanov D. Breast MRI, digital mammography and breast tomosynthesis: comparison of three methods for early detection of breast cancer. Bosn J Basic Med Sci. 2015 Nov; 15(4): 64-8.

15 Skaane P, Bandos AI, Gullien R, Eben EB, Ekseth U, Haakenaasen U, et al. Comparison of digital mammography alone and digital mammography plus tomosynthesis in a population-based screening program. Radiology. 2013 Apr;267(1):47-56.

16 Lourenco AP, Barry-Brooks M, Baird GL, Tuttle A, Mainiero MB. Changes in recall type and patient treatment following implementation of screening digital breast tomosynthesis. Radiology. 2015 Feb;274(2):337-42.

17 Sharpe RE Jr, Venkataraman S, Phillips J, Dialani V, Fein-Zachary VJ, Prakash S, et al. Increased cancer detection rate and variations in the recall rate resulting from implementation of 3D digital breast tomosynthesis into a population-based screening program. Radiology. 2016 Mar;278(3):698-706.

18 Gilbert FJ, Tucker L, Gillan MG, Willsher P, Cooke J, Duncan KA, et al. Accuracy of digital breast tomosynthesis for depicting breast cancer subgroups in a UK retrospective reading study (TOMMY trial). Radiology. 2015 Dec;277(3):697-706.

19 Refaat R, Matar MM. Digital breast tomosynthesis compared to digital mammography in a series of Egyptian women with pathologically proven breast cancer. Egypt J Radiol Nucl Med. 2015;46(4):1241-8.

20 Sickles EA, D’Orsi CJ, Bassett LW, et al. ACR BI-RADS Mammography. In: ACR BI-RADS Atlas, Breast Imaging Reporting and Data System. 5th ed. Reston (VA): American College of Radiology; 2013. p. 134-6.
21 Park SH, Goo JM, Jo CH. Receiver operating characteristic (ROC) curve: practical review for radiologists. Korean J Radiol. 2004 JanMar;5(1):11-8.

22 Altman DG. Practical statistics for medical research. London: Chapman and Hall; 1991.

23 Rafferty EA, Durand MA, Conant EF, Copit DS, Friedewald SM, Plecha DM, et al. Breast cancer screening using tomosynthesis and digital mammography in dense and nondense breasts. JAMA. 2016 Apr;315(16):1784-6.

24 Park JM, Franken EA Jr, Garg M, Fajardo LL, Niklason LT. Breast tomosynthesis: present considerations and future applications. Radiographics. 2007 Oct;27 Suppl 1:S231-40.

25 Kopans DB. Digital breast tomosynthesis: a better mammogram. Radiology. 2013 Jun; 267(3):968-9.

26 Spangler ML, Zuley ML, Sumkin JH, Abrams G, Ganott MA, Hakim C, et al. Detection and classification of calcifications on digital breast tomosynthesis and 2D digital mammography: a comparison. AJR Am J Roentgenol. 2011 Feb;196(2):320-4.

27 Svahn TM, Houssami N, Sechopoulos I, Mattsson S. Review of radiation dose estimates in digital breast tomosynthesis relative to those in two-view full-field digital mammography. Breast. 2015 Apr;24(2):93-9.

28 Alkhalifah K, Brindhaban A. Investigation of Exposure Factors for Various Breast Composition and Thicknesses in Digital Screening Mammography Related to Breast Dose. Med Princ Pract. 2018;27(3):211-6.

29 Skaane P. Breast cancer screening with digital breast tomosynthesis. Breast Cancer. 2017 Jan;24(1):32-41.

30 Lobbes MB, Houben I. Contrast-enhanced tomosynthesis: the best of both worlds or more of the same? Eur J Radiol. 2016 Feb; 85(2):507-8. 